\title{
Testosterone replacement therapy (TRT) and its effect on bone marrow. How serious is it and is there a true polyglobulia?
}

\author{
Levcikova $\mathrm{M}^{1}$, Breza $\mathrm{Jr}^{2}$, Luha $\mathrm{J}^{3}$, Dubravicky $\mathrm{J}^{5}$, Kovacova $\mathrm{E}^{4}$, Fillo $\mathrm{J}^{5}$ \\ Department of Urology, University Hospital Bratislava, Slovakia. miskalevcikova@gmail.com
}

\begin{abstract}
INTRODUCTION: TRT in men with testosterone deficiency syndrome (TDS) had multiple positive effects and restore a quality of life of affected men. Polyglobulia is the most common dose-limiting adverse effect of TRT, but the mechanisms of TRT-mediated erythropoesis remain unclear. In this study, we evaluated long term haematological side effects of TRT: polyglobulia, elevated hemoglobin $(\mathrm{Hb})$ and haematocrit $(\mathrm{Ht})$.

METHODS: In a cross-sectional descriptive study, the authors treated 69 men with TDS and the average age 59 years and the follow-up period 81.32 months. The men were treated with three-month i.m. injections of 1000 $\mathrm{mg}$ testosterone undecanoate. The elevated values were: $\mathrm{Hb}$ above $176 \mathrm{~g} / \mathrm{l}, \mathrm{Ht}$ above 0.52 and erythrocytes (Ery) above $6.0 \mathrm{mil} / \mathrm{mcl}$.

RESULTS: 21 out of 69 patients (30.43\%) had an increased $\mathrm{Hb}, \mathrm{Ht}$ or Ery during treatment. The interesting fact was that only five men ( $7.24 \%)$ had increased the number of Ery (true polyglobulia). No men with elevated level of $\mathrm{Hb}, \mathrm{Ht}$ or Ery had other side effects (like thrombosis).

CONCLUSION: It is still not clear, why in some men on TRT the feedback does not work and bone marrow production of red blood cells continues even if the upper limit is reached. Authors expect that only $7 \%$ of men had true polyglobulia, other men had elevated $\mathrm{Hb}$ or $\mathrm{Ht}$. Based on our own experience we recommend a regular check of men on TRT on order to avoid possible serious side-effects (Tab. 1, Fig. 2, Ref. 25). Text in PDF www.elis.sk. KEY WORDS: testosterone, testosterone replacement therapy, hemoglobin, polyglobulia, haematocrit.
\end{abstract}

\section{Introduction}

TRT of men with TDS has many beneficial effects. Overall health condition and function of many organ systems are improved significantly, among them the bone marrow function. Consequently, blood count began to improve. However, some risks have to be considered. One of the risks of TST replacement therapy is polyglobulia and hematocrit increase occurring in 4-40\% of men on TRT, and may worsen pre-existing cardiovascular complications secondary to an increased blood viscosity $(1,2)$. It is still not clear, why in some men on TRT feedback does not work and bone marrow production continues even if the upper limit of $\mathrm{Hb}$, Ery, $\mathrm{Ht}$ was reached. Many studies described polyglobulia and mentioned only $\mathrm{Hb}$ and $\mathrm{Ht}$, and not Ery (3).

\section{Materials and methods}

The authors treated 69 men with TDS after a complete urology and internal examination. They did not suffer from other serious

${ }^{1}$ Department of Urology, University Hospital Bratislava, Slovakia, ${ }^{2}$ Department of Pediatric Urology, University Hospital Bratislava, Slovakia, ${ }^{3}$ Institute of Medical Biology, Genetics and Clinical Genetics, School of Medicine, Comenius University Bratislava, Slovakia, ${ }^{4}$ Department of Internal Medicine I, Comenius University Bratislava, Slovakia, and ${ }^{5}$ Urology Clinic, University Hospital Bratislava, Slovakia

Address for correspondence: J. Fillo, MD, Urology Clinic, University Hospital Bratislava, Limbova 5, SK-833 05 Bratislava, Slovakia. urological condition, mainly from nutrition problems. None of these men had an obstructive pulmonary disease or other serious internal comorbidity. The average follow-up time was 81.32 months. The mean age was 59 years. The men were treated with three-month i.m. injections of 1000mg testosterone undecanoate.

During the treatment, all men had testosterone (TST) level within the physiological range. The men were regularly checked in accordance with the EAU guidelines. The elevated values are (according to laboratory): hemoglobin above $176 \mathrm{~g} / \mathrm{l}$, hematocrit above 0.52 and erythrocytes above $6.0 \mathrm{mil} / \mathrm{mcl}$. None of these men had an obstructive pulmonary disease or other serious internal comorbidity.

As stated in the EAU guidelines 2017, technique of blood collection is important. There should be minimal or no venous occlusion during the blood sampling.

\section{Results}

In the first year, the treatment hemoglobin rose from 151.93 $\mathrm{g} / \mathrm{l}$ to $161.93 \mathrm{~g} / \mathrm{l}$ and then it remained stable (Fig. 1). In 21/69 (30.43 \%) men, some of the studied parameters (Ery, Hb, Ht) increased above the normal range (Tab. 1). Only five men (7.24 \%) had $\mathrm{N}$ increased number of Ery. The others had only increased $\mathrm{Hb}$ and Ht. The size of Ery was not enlarged (Fig. 2). These elevated values were not only at the beginning, but also during the treatment. The men with THE elevated level of Hb, Ht or Ery had no vascular complications. In the patients with supranormal levels 


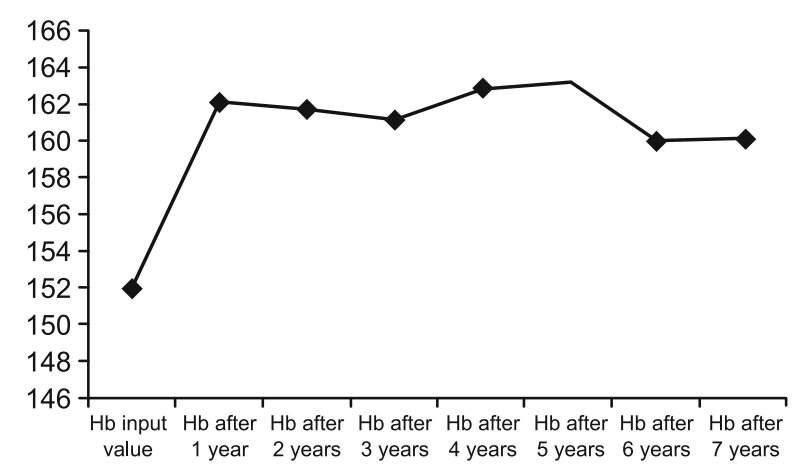

Fig. 1. The monitored level of hemoglobin.

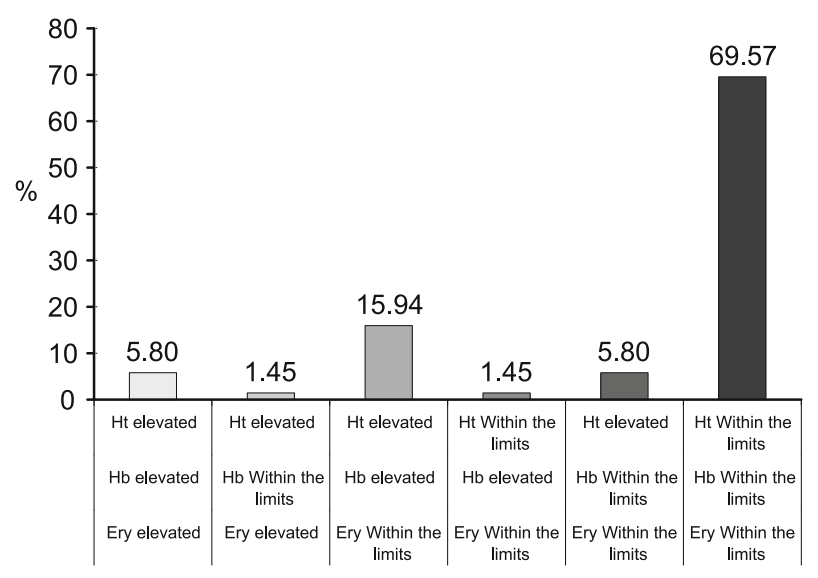

Fig. 2. Distribution of elevated Ery, Hb, Ht.

of the $\mathrm{Hb}, \mathrm{Ht}$ and Ery, acetylsalicylic acid $30 \mathrm{mg}$ per os daily was recommended.

\section{Discussion}

Testosterone is an anabolic hormone and besides influencing different organ systems, it stimulates also bone marrow. In some patients, the red blood components increase during the treatment. Authors proved a correlation between high testosterone levels and high hemoglobin, because testosterone stimulates erythropoesis. Erythrocytosis can develop during testosterone treatment, especially in older men, treated by injectable testosterone preparations (4). Aghasadeh et al 2015 recommended monitoring of dihydrotestosterone level, which can be responsible for polyglobulia. They recommended therapeutic use of 5ARIs, which may inhibit the conversion of TST to dihydrotestosterone. We tried to use 5ARI (finasterid) in one men with polyglobulia and symptomatic BPH, but without a success. The testosterone-treated men were at a higher risk of developing erythrocytosis than the placebo/nonintervention group. The adverse effects of testosterone therapy include an increase in hemoglobin and hematocrit (5). A meta-analysis of 51 studies revealed that TRT is associated with an average $0.8 \mathrm{~g} / \mathrm{dl}$ increase in $\mathrm{Hb}$ and a $3.2 \%$ increase in $\mathrm{Ht}$, but there was no significant effect on mortality, prostate size or
Tab. 1. Elevation of Ht, $\mathrm{Hb}$ and Ery.

\begin{tabular}{lllrr}
\hline Erythrocytes & Hemoglobin_ & Hematocrit & $\mathrm{n}$ & $\%$ \\
Ery elevated & Hb elevated & Ht elevated & 4 & 5.80 \\
Ery elevated & Hb normal & Ht elevated & 1 & 1.45 \\
Ery normal & Hb elevated & Ht elevated & 11 & 15.94 \\
Ery normal & Hb elevated & Ht normal & 1 & 1.45 \\
Ery normal & Hb normal & Ht elevated & 4 & 5.80 \\
Ery normal & Hb normal & Ht normal & 48 & 69.57 \\
\hline & & Summary & $\mathbf{6 9}$ & 100.00 \\
\hline
\end{tabular}

cardiovascular outcomes (5). It is not exactly known, which level of $\mathrm{Ht}$ is associated with adverse health events. In epidemiologic studies, higher Ht levels are associated with an increased risk of hypertension, cardiovascular events and stroke than lower $\mathrm{Ht}$ values $(6,7)$. As Ht levels increase from low to normal levels, plasma viscosity and tissue blood flow increase along with an increase in tissue oxygen delivery (8-11). However, as hematocrit levels rise further, plasma viscosity increases disproportionately, and at some level of hematocrit, tissue blood flow and oxygen delivery begin to decline (8-11). The Endocrine Society's expert panel recommends that testosterone administration should be withheld in men, whose hematocrit rises above $54 \%$ during testosterone therapy. When the hematocrit has fallen into the normal range, TRT can be reinstituted at a lower dose (12). The frequency of neuro-occlusive events (stroke) in association with an increased hematocrit in testosterone trials has been extremely low (13).

Men have higher levels of hemoglobin than women, and hypogonadism causes a decline in $\mathrm{Hb}$ levels that can be restored with testosterone replacement therapy $(14,15)$. However, the elevation in haemoglobin above certain levels may lead to complications, particularly in elderly, because the increase in blood viscosity could exacerbate vascular disease in the coronary, cerebrovascular, or peripheral vascular circulation, especially in people with other diseases that cause secondary polycythemia, i.e. chronic obstructive pulmonary disease (18-20).

Injection of TST is associated with a higher potential for erythrocytosis than application of local (topic) preparations (21). Periodic hematological assessment is indicated (i.e., before treatment, then in 3 to 4 months and in 12 months in the first year of treatment and annually thereafter). While it is not yet clear, what critical threshold is a desirable, dose adjustment and/or periodic phlebotomy may be necessary to keep hematocrit below $52 \%$ to $55 \%$ (22).

Erythrocytosis, or polycythemia, is a known side effect of testosterone treatment. A meta-analysis of adverse effects of TST treatment in men with TDS found 11 trials that highlighted erythrocytosis as a prominent side effect of treatment with TST. However, the mechanism behind the causes of hemoconcentration, and how this may affect men, is poorly understood (5). Covioelo et al (2008) studied elevated hemoglobin and hematocrit in patients receiving TST treatment. They demonstrated that treatment with testosterone caused statistically significant increased hemoglobin levels $(0.86 \pm 0.31 \mathrm{~g} / \mathrm{dl}, \mathrm{p}=0.01)$. The authors hypothesized that treatment with TST increased serum erythropoetin, leading 
to erythrocytosis, yet this was disproven $(-0.24 \pm 2.16 \mathrm{mIU} / \mathrm{ml}$, p $=0.91)$. Another proposed theory posits that TST has a dose dependent stimulatory effect on erythropoesis in men that is more pronounced in older men.

Li et al (2016) studied 102,650 exogenous testosterone treated and 102,650 untreated patients. They found no significant association between the exogenous testosterone therapy and incidents of idiopathic or overall venous thrombotic events in men with hypogonadism. However, some discrepant findings exist for the association between the injectable formulations and the risk of overall venous thrombotic events.

Snyder et al (1999) noticed that erythrocytosis occurred in 5.5 $\%$ of scrotal transdermal users, and the majority of changes took place over the first three months of treatment (24). The frequency of polycythemia (hematocrit over $51 \%$ ) was related mainly to supra-physiological serum TST levels.

Testosterone increases erythropoetin levels, but the erythropoetin levels return towards baseline with a continued testosterone therapy (23). However, erythropoetin levels are elevated in relation to the increased haemoglobin levels in testosterone-treated men. Testosterone might potentially increase the sensitivity of erythroid progenitor cells to erythropoetin.

In another study, within the period of 36 months, Maggio et al (2013) reported, that there were no 'serious' patient-centered adverse events (e.g. cerebrovascular accident, vascular occlusive events, venous thromboembolisms).

There are many TDS-treated men with TST worldwide. The side effects of this treatment are also polycytemia, increase of hemoglobin and hematocrit. The increments in hemoglobin and hematocrit are related to testosterone dose and concentrations and occur more frequently in men aged $60-75$ years, than in men aged 19-35 years (23).

Increasing age was associated with increasing odds for the development of polycythemia in androgen deficient men treated with testosterone. Physiological experiments, when normal men are rendered acutely with androgen deficit and then treated with graded and matched doses of TST, confirm this age association (23).

Although TRT in androgen-deficient men clearly stimulates erythrocytosis and increases the odds of polycythemia fourfold (13), the mechanism by which this occurs, remains elusive.

Physiological experiments in normal men do not show the relationship between TST dose and immunoreactive erythropoetine after five months of treatment (23). Ip et al (2010) conclude, that higher through serum testosterone levels, but not duration of treatment predict the development of polycythemia in men receiving long-acting depot TST treatment. These data inform the clinical practice. Further investigation of the mechanism by which polycythemia develops is required (25).

\section{Conclusion}

The anabolic effect of testosterone has many positive effects, including bone marrow stimulation and improvement in blood count as we documented in our patients. This helps to improve tissue supply with oxygen and reduces fatigue. The authors found that not all components of blood count are elevated. They found only $7 \%$ of men with polyglobulia (elevated erythrocytes count) and the rest had elevated $\mathrm{Hb}$ and $\mathrm{Ht}$. No men with elevated blood count had thrombotic complications. Further studies will be needed to clarify, why in some men feedback does not work and bone marrow continues to increase the production of haemoglobin. It is necessary to check the blood count at regular intervals. In these cases, it is a simple solution to extend the administration interval of TST from 12 to 14 weeks or more to eliminate the potential risk resulting from an increased blood viscosity.

\section{References}

1. Rhoden EL, Morgentaler A. Risks of Testosterone-Replacement Therapy and Recommendations for Monitoring. N Engl J Med. 2004; 350: 482.

2. Bassil N, Alkaade S, Morley JE. The benefits and risks of testosterone replacement therapy: a review. Ther Clin Risk Manag 2009; 5: 427.

3. Aghazadeh M, Pastuszak AW, Johnson WG, McIntyre MG, Hsieh TM, Lipshultz LI. Elevated Dihydrotestosterone is Associated with Testosterone-induced Erythrocytosis. J Urol 2015; 194 (1): 160-165.

4. Wang $C$, Nieschlag $E$, Swerdloff $R$ et al. Investigation, treatment and monitoring of late-onset hypogonadism in males: ISA, ISSAM, EAU, EAA and ASA recommendations. Eur J Endocrinol 2008; 159: 507-514.

5. Fernández-Balsells MM et al. Clinical review 1: Adverse effects of testosterone therapy in adult men: a systematic review and meta-analysis. J Clin Endocrinol Metab 2010; 95: 2560-2575.

6. Strand A et al. Increased hematocrit before blood pressure in men who develop hypertension over 20 years. J Am Soc Hypertens 2007; 1: 400-406.

7. Gagnon DR, Zhang TJ, Brand FN, Kannel WB. Hematocrit and the risk of cardiovascular disease -the Framingham study: a 34-year followup. Am Heart J 1994; 127: 674-682.

8. Hagl S et al. The effect of hemodilution on regional myocardial function in the presence of coronary stenosis. Basic Res Cardiol 1977; 72: 344-364.

9. Danesh J, Collins R, Peto R, Lowe GD. Hematocrit, viscosity, erythrocyte sedimentation rate: meta-analyses of prospective studies of coronary heart disease. Eur Heart J 2000; 21: 515-520.

10. Burch GE, DePasquale NP. Hematocrit, viscosity and coronary blood flow. Dis Chest 1965; 48: 225-232.

11. Woodward M, Rumley A, Tunstall-Pedoe H, Lowe GD. Does sticky blood predict a sticky end? Associations of blood viscosity, hematocrit and fibrinogen with mortality in the West of Scotland. Br J Haematol 2003; 122: 645-650.

12. Bhasin $\mathrm{S}$ et al. Testosterone therapy in men with androgen deficiency syndromes: an Endocrine Society clinical practice guideline. J Clin Endocrinol Metab 2010; 95: 2536-2559.

13. Calof $\mathbf{O M}$ et al. Adverse events associated with testosterone replacement in middle-aged and older men: a meta-analysis of randomized, placebo-controlled trials. J Geront A Biol Sci Med 2005; 60: 1451-1457.

14. Sih R, Morley JE, Kaiser FE, Perry III HM, Patrick P, Ross C. Testosterone replacement in older hypogonadal men: a 12 months randomized controlled study. J Clin Endocrinol Metab 1997; 82: 1661-1667.

15. Basaria S, Dobs AS. Risks versus benefits of testosterone therapy in elderly men. Drugs Aging 1999; 15: 131-142. 
16. Viallard JF, Marit G, Mercie P, Leng B, Reiffers J, Pellegrin JL. Polycythaemia as a complication of transdermal testosterone therapy. $\mathrm{Br}$ J Haematol 2000; 110: 237-238.

17. The Endocrine Society. Clinical bulletins in andropause: benefits and risks of treating hypogonadism in the aging male. Endocr Rep 2002; 2: 1-6.

18. Kim YC. Testosterone supplementation in the aging male. Testosterone supplementation in the aging male. Int J Impot Res 1999; 11: 343-352.

19. Dobs AS, Meikle AW, Arver S, Sanders SW, Caramelli KE, Mazer NA. Pharmacokinetics, efficacy, and safety of a permeation-enhanced testosterone transdermal system in comparison with bi-weekly injections of testosterone enanthate for the treatment of hypogonadal men. J Clin Endocrinol Metab 1999; 84: 3469-3478.

20. Bassil et al, publisher and licensee Dove Medical Press Ltd. This is an Open Access article which permits unrestricted non commercial use, provided the original work is properly cited. Ther Clin Risk Manag 2009; 5: 427-448.

21. Li H, Benoit K, Wang W, Motsko S. Association between Use of Exogenous Testosterone Therapy and Risk of Venous Thrombotic Events among Exogenous Testosterone Treated and Untreated Men with Hypogonadism. J Urol 2016; 195 (4P1): 1065-1072. Doi:10.1016/j.juro. 2015.10.134. Epub 2015 Oct 31.

22. Snyder PJ, Peachey H, Hannoush P, Berlin JA, Loh L, Lenrow DA. Effect of testosterone treatment on body composition and muscle strength in men over 65 years of age. J Clin Endocrinol Metab 1999; 84: 2647-2653.

23. Coviello A, Kaplan B, Lakshman K, Chen T, Singh A, Bhasin S. Effects of graded doses of testosterone on erythropoiesis in healthy young and older men. J Clin Endocrinol Metab 2008; 93: 914-919.

24. Maggio M, Snyder P, Ceda G, Milaneschi Y, Luci M, Cattabiani C et al. Is the haematopoietic effect of testosterone mediated by erythropoietin? The results of a clinical trial in older men. Andrology 2013; 1: 24-28.

25. Ip FF, Pierro I, Brown R, Cunningham I, Handelsman DJ, Liu PY. Through serum testosterone predict the development of polycythemia in hypogonadal men treated for up to 21 years with subcutaneous testosterone pellets. Eur J Endocrin 2010; 162: 185-390.

Received June 27, 2017. Accepted August 4, 2017. 